\title{
Tecnociencia: una innovación educativa enemiga del pasado
}

\author{
Technological Education: \\ An Educational Innovation Enemy of the Past
}

\author{
Emgelberth Vargas \\ emgelberth@gmail.com n https://orcid.org/0000-0003-3098-0719 \\ Doctor en Ciencias de la Educación \\ Universidad Bicentenaria de Aragua, Venezuela
}

Fecha de recepción: 31 de marzo de 2020

Fecha de aceptación: 23 de abril de 2020

Fecha de publicación: 30 de junio de 2020

\section{RESUMEN}

El siglo XXI ha representado una era de cambios, así como de nuevas maneras de ver o percibir las realidades desde las miradas de los otros. Es así que se presentan nuevos paradigmas y parte de ello se refleja en las innovaciones tecnológicas que se hacen perentorias en cada uno de los ámbitos donde se desarrolla la sociedad. En este sentido emerge la educación tecnocientífica (ETCS), como una de esas innovaciones que se configuran en una necesidad para las representaciones sociales de los actores educativos en el contexto universitario. La ETCS se refiere al conjunto de disciplinas que se engranan para estudiar aspectos sociales de la ciencia y tecnología, concebidas como áreas indispensables en el aquí y ahora, convirtiéndose en el eje central para poder desarrollar en las personas una cultura tecnocientífica.

Este artículo posee como eje teleológico central, develar los fundamentos explicativos de las representaciones sociales de los actores educativos, acerca de la alfabetización tecnocientífica en el contexto universitario de la Universidad Nacional Pedro Henríquez Ureña(UNPHU) en Santo Domingo, República Dominicana. La metodología se estructura desde la episteme fenomenológica hermenéutica. Para ello, se seleccionarán tres actores educativos pertenecientes al Departamento de Ciencias. La obtención de la información se hará a través del análisis documental y la técnica de la entrevista en profundidad, que posee como instrumento al guión de dicha entrevista. Se pudo conocer que desde los pensamientos y representaciones sociales de los actores educativos, se edifican fundamentos que denotan la relevancia de propiciar en el contexto universitario la educación tecnocientífica; y con ello la alfabetización de modo imperante en una era donde la ciencia y la tecnología deben ser apoyadas desde los entes gubernamentales, para poder propiciar el desarrollo de un país.

Palabras clave: representaciones sociales, educación tecnocientífica, cultura tecnocientífica. 


\begin{abstract}
The 21st century has represented an era of changes as well as new ways of seeing or perceiving realities from the eyes of others, therefore new paradigms are presented and part of it is reflected in the technological innovations that become peremptory in each one of the areas where society develops. In this regard, techno-scientific education (ETCS) emerges, as one of those innovations that are configured as a necessity for the social representations of educational actors in the university context, the ETCS refers to the set of disciplines that are geared to study social aspects of science and technology conceived as indispensable areas in the here, in the now, becoming the central axis to develop in people a techno-scientific culture. This article has as its central teleological axis to reveal the explanatory foundations of the social representations of educational actors about techno-scientific literacy in the university context of the Universidad Nacional Pedro Henríquez Ureña, UNPHU, in Santo Domingo, Dominican Republic. The methodology is structured from the hermeneutical phenomenological spirit. For this, three (3) educational actors belonging to the Science Department will be selected. The information will be obtained through documentary analysis and the in-depth interview technique that the interview script uses as an instrument. It was learned that from the thoughts and social representations of educational actors, foundations are built that denote the relevance of promoting technoscientific education in the university context and with it, literacy prevailing in an era where science and technology must be supported. from government entities to enable the development of a country.
\end{abstract}

Keywords: Social Representations, Technoscientific Education, Technoscientific Culture.

\section{Introducción}

no es hacer avanzar el conocimiento,

sino saber»

Serge Moscovici

En la actualidad han surgido innovaciones educativas que facilitan de una manera $u$ otra acentuar el conocimiento en los estudiantes; sin embargo, más que fomentar nuevos procesos pedagógicos se necesita que estas innovaciones originen cambios socioculturales que impliquen un desarrollo para el país. Para nadie es un secreto que las sociedades se encuentran en constante transformación y por ello se busca concebir su formación desde el contexto educativo transdisciplinario. En este sentido, la presente investigación busca enfatizar la importancia de la educación tecnocientífica desde las representaciones sociales de los actores educativos que hacen vida en el Departamento de Ciencias de la Universidad Nacional Pedro Henríquez Ureña (UNPHU) en Santo Domingo,
República Dominicana. El propósito es que se promueva la aplicación del conocimiento científico de las ciencias básicas en favor de una culturización tecnocientífica.

Para ello es elemental hacer referencia a actores educativos, como grupos sociales que comparten un conocimiento con significados cognitivos basados en el rigor científico. Reconocidas como representaciones sociales según la Teoría de Moscovici (1981) y Jodelet (1988); asímismo se aborda la acción discursiva de estos actores educativos como los modos de vida que se configuran en la construcción de un conocimiento comunitario o pensamiento social conocido como construccionismo social por Kenneth Gergen.

De esta manera se pretende hacer énfasis en la relación de las representaciones sociales y el construccionismo social desde el contexto universitario en un ámbito educativo, donde la cultura tecnológica y científica se unen con la tecnociencia para llevar a cabo las innovaciones pedagógicas que trae consigo la educación 
tecnocientífica; así como la ruptura paradigmática que emerge desde la cosmovisión de la tecnología como medio para aplicar, desarrollar y transmitir las ciencias básicas en pleno siglo XXI.

\section{Transitando la realidad contextualizada}

«Puede que actualmente haya otros conocimientos que adquirir, otras cuestiones que plantearse, partiendo, no de lo que los demás han conocido, sino de lo que han ignorado».

Serge Moscovici

En pleno siglo XXI, tener una definición única de sociedad es imposible, pues esta cada vez se vuelve más compleja ya que se configura desde los diversos contextos y modos de vida de los actores sociales que la componen. Lo cierto es que su evolución implica nuevas maneras de obtener el conocimiento que parte de cubrir las necesidades de una comunidad, en este caso científica, con el uso del pensamiento crítico reflexivo. Por ello se genera conocimiento con interrelación de las cosmovisiones que se posee de las ciencias básicas como disciplinas, que aún segmentadas, pueden develar la importancia de un fenómeno desde su engranaje.

Cabe destacar que la segmentación que se ha señalado se refiere al estudio y aplicación de la química, física y matemáticas desde el conocimiento científico; no obstante, es este mismo conocimiento el que se complementa con otras áreas debido a la necesidad imperante de innovar. Es de este modo como emerge la tecnociencia en las últimas décadas, facilitando la construcción del conocimiento científico desde un complejo entramado de la tecnología en las ciencias. Esta es promovida incluso por la UNESCO, en su esfuerzo de poner en marcha el desarrollo sostenible de sus países miembros.

En efecto, la tecnociencia es reconocida como la sociedad del conocimiento por hacer uso de las tecnologías de la información y la comunicación, como el medio idóneo de transmisión del conocimiento científico en pro de crear nuevos imaginarios sociales en el ámbito educativo; en el modo de educar e implementar herramientas y técnicas en los procesos pedagógicos con visión de progreso. No obstante, aún se debe alfabetizar a la sociedad con el uso de estas herramientas.

En el mismo orden de ideas, se debe acotar que la educación tecnocientífica permite desarrollar en la sociedad una manera distinta de apreciar el mundo, resignificando el valor de los fenómenos que han permitido hacer vida en el planeta Tierra. Es por ello que se busca hacer énfasis en la educación tecnocientífica como innovación educativa y tecnológica, llámese también como tecnología educativa desde las ciencias. De este modo se desarrolla en la sociedad uno de los aspectos más esenciales, como lo es el educativo, para obtener una mejor calidad de vida.

No es un secreto para la sociedad, que el mundo se encuentra en proceso de regeneración de la naturaleza debido a fenómenos que han provocado cambios vertiginosos en el modo de vivir y accionar de las personas; por ello se hace obligatorio una formación educativa basada en el desarrollo de las capacidades tecnológicas en los actores educativos, dando así cabida a la necesidad de que exista una alfabetización tecnocientífica. Por tal motivo, Sunkel y Trucco (2013), sostienen que la tecnología debe tener mayor presencia en los contextos educativos, ya que se relaciona directamente con el auge y desarrollo de un país a partir del aprendizaje de las competencias del siglo XXI.

Lo que los autores quieren decir es que, actualmente, se deben desarrollar habilidades cognitivas a partir de las competencias tecnológicas que brinda la sociedad del conocimiento, como parte de una alfabetización cultural de los nuevos tiempos. En este sentido, la educación tecnocientífica se constituye como una 
competencia medular para poder cubrir las exigencias de la sociedad en cuanto a las habilidades pedagógicas, que debe desarrollarse desde la gestión tecnológica en las ciencias básicas.

Al respecto, se concibe el contexto universitario como substancia central en la producción del conocimiento científico y epicentro de las nuevas cosmovisiones de la ontología digital. Es desde el paradigma tecnológico donde se busca estructurar y direccionar el impacto de los aprendizajes que se entraman en un enfoque posmoderno de una sociedad transformacional y transdisciplinaria. Es evidente que la educación tecnocientífica mitiga las barreras de tiempo y espacio como una epistemología emergente de las competencias pedagógicas que orientan a la educación hacia nuevos modos y medios de aprender y aplicar el conocimiento científico.

En función a lo expuesto se busca comprender las cosmovisiones existentes respecto al abordaje de las ciencias básicas mediante las tecnologías, con la implementación de la educación tecnocientífica desde los actores educativos, para poder obtener fundamentos explicativos sobre su alfabetización en el contexto de la UNPHU.

Sobre estos argumentos la intención es develar los fundamentos obtenidos para evidenciar la necesidad de alfabetización tecnocientífica que impera en el siglo XXI, en una sociedad que se encuentra en plena transformación de pensamiento y cultura ante las adversidades que hoy día nos arropan. Es por ello que surgen las siguientes interrogantes:

¿Cómo es el tipo de cultura tecnológica que se ha configurado en los estudiantes del Departamento de Ciencias de la UNPHU?

¿Cuáles serían las patologías del desarrollo social en el mundo universitario, con respecto al abordaje de la tecnociencia en países en vías de desarrollo sostenible?

¿Cómo representan la tecnociencia los nativos e inmigrantes digitales de la UNPHU Santo Domingo?
¿Cómo se presenta la alfabetización de la tecnociencia en aras del desarrollo sostenible, desde la intersubjetividad de los propios actores, en el contexto universitario de la UNPHU en Santo Domingo?

\section{Ejes teleológicos}

Eje peleológico principal

-Develar los fundamentos explicativos de las representaciones sociales de los actores educativos, acerca de la alfabetización tecnocientífica, en el contexto universitario de la UNPHU.

\section{Ejes teleológicos específicos}

-Diagnosticar la cultura tecnológica que se ha configurado en los actores educativos del Departamento de Ciencias de la UNPHU.

-Interpretar la cosmovisión de los actores educativos con respecto a la cultura tecnológica en las ciencias básicas en el contexto de la UNPHU.

- Construir una red de relaciones de la noción de realidad sobre la educación tecnocientífica aprehendida a partir de saberes, interpretaciones y acciones desde la intersubjetividad de los propios actores en el contexto universitario de la UNPHU.

\section{Fundamentos} epistemológicos

«¿Por qué esta magnífica tecnología científica, que ahorra trabajo y nos hace la vida más fácil, nos aporta tan poca felicidad? La respuesta es esta: simplemente porque aún no hemos aprendido a usarla con tino».

Albert Einstein

\section{Las representaciones sociales y el construccionismo social}

En todo contexto educativo se pueden percibir diversos modos de comunicación que se relacionan de una manera $\mathrm{u}$ otra con el conocimiento que han de transmitir los individuos mediante su comportamiento en la interacción social. Este modo de comunicación 
es reconocida por Moscovici (1979) citado en Mora $(2002 ; 7)$ como representación social y se comprende como:

La representación social es una modalidad particular de conocimiento, cuya función es la elaboración de los comportamientos y la comunicación entre los individuos. La representación es un corpus organizado de conocimientos y una de las actividades psíquicas gracias a las cuales, los hombres hacen inteligible la realidad física y social, se integran en un grupo o en una relación cotidiana de intercambios, liberan los poderes de su imaginación.

Según el autor, cada compendio de conocimiento que se comparte en los modos de vida de una sociedad se denomina «representaciones sociales». Estas van a develar una simbología desde las interpretaciones que emergen del psicoanálisis, el público y la imagen. En este sentido, en las universidades se contempla un universo de representaciones sociales que va a depender del conocimiento que estos actores sociales posean respecto a un fenómeno determinado.

En función del auge tecnológico que se ha desarrollado con el devenir de los años, el modo de transmitir el conocimiento y de comunicarse también experimenta cambios que van a depender del conocimiento que se maneja en una comunidad en específico. En efecto, el conocimiento que se comparte en los grupos sociales como los actores educativos del Departamento de Ciencias en la UNPHU en Santo Domingo, se fundamenta en el conocimiento científico y su comprobación con el abordaje de las ciencias básicas. Esto significa que existe una relación entre lo que transmite y su significado, tal como lo que afirma Robert Farr $(1983 ; 665)$ son «sistemas cognitivos con lógica y lenguaje propio». Pero esta codificación que se produce en cuanto al modo de obtener el conocimiento, se ve influenciada en la actualidad por la aplicación de estas interacciones cognitivas: tecnología-ciencia-sociedad.

Es por ello que se hace mención de las representaciones sociales como los sistemas cognitivos que permiten, a través del pensamiento práctico, interpretar y comprender los constructos o ideales que configuran a los actores sociales dentro de un determinado contexto, tal como lo expresa Jodelet (1988), citado por Vergel (2016; 35), son: «sistemas de referencia que nos permiten interpretar lo que nos sucede, e incluso dar un sentido a lo inesperado; formas de conocimiento social que permiten interpretar la realidad cotidiana». Esto quiere decir que ese constructo cognitivo define a una sociedad. En tal caso, se identifican a los docentes o actores educativos del Departamento de Ciencia de la UNPHU, como representaciones sociales.

Por su parte, Kanneth Gergen (1996) sostiene a través del construccionismo social, que existe una línea muy delgada entre comunicar y transmitir el conocimiento, y esta tiene que ver con la cultura. Hoy día puede hablarse de la cultura tecnológica ya que surge de la necesidad de la sociedad de desarrollar competencias para poder establecer esa comunicación e interacción de constructos cognitivos desde una epistemología social con rigor científico.

Con base en lo sostenido, los constructos que emergen de la acción discursiva en las representaciones sociales de los actores educativos del Departamento de Ciencias de la UNPHU, se consideran como un construccionismo social.

\section{Cultura tecnocientífica}

El término de tecnociencia fue usado por primera vez por Bruno Latour en 1983, para abreviar la denominación «ciencia y tecnología», significando para la actualidad la fusión de los mismos. Ahora bien, la necesidad de actualización constante que caracteriza el siglo XXI suma a la «sociedad» como una triada que se complementa debido a que cada proceso de innovación genera cambios en la cosmovisión existente entre tecnología-ciencia-sociedad. Partiendo de esta premisa, la tecnología permite el desarrollo de la ciencia que es abordada por y para la sociedad, acercándose a través de la cultura científica al modo de obtener 
un conocimiento y aplicarlo. Es así como lo plantea Pickering (1992): la cultura científica es «el paso de la ciencia como conocimiento de la ciencia como práctica y cultura». Para este autor, este enfoque cultural da paso a una alfabetización tecnocientífica, donde el conocimiento connota la adaptación del hombre a la tecnología.

La triada ciencia-tecnología-sociedad permite el desarrollo del pensamiento científico para generar productos cognitivos y constructivos en una era donde las innovaciones educativas son esenciales. En este orden de ideas, se vincula a los actores educativos del Departamento de Ciencias de la UNPHU en Santo Domingo, con la necesidad de llevar a cabo una alfabetización tecnocientífica inducida por las innovaciones tecnológicas con respecto a la comprobación del conocimiento científico en las ciencias básicas. Por lo tanto, es menester hacer énfasis en el desarrollo de una cultura tecnocientífica como el enfoque hacia donde se dirige la sociedad del conocimiento en pleno siglo XXI.

Para finalizar, es notoria la necesidad de insertar la cultura tecnocientífica en el ámbito educativo como una alfabetización tecnocientífica que posibilite la comprensión y la aceptación del conocimiento científico de la mano de las tecnologías; como una innovación que trae para la sociedad la implementación de las investigaciones y la puesta en marcha de proyectos beneficiosos para el desarrollo del país.

\section{Bases legales}

\section{Ley 66-97 Ley General de Educación}

La Ley General de la Educación de República Dominicana reza en su artículo 8, sobre las competencias del Estado y sus funciones en materia de educación, ciencia y cultura:

a) Fortalecer y mejorar la enseñanza de la ciencia y la tecnología en todos los niveles educativos, educando para el mejor uso de ellas y para evitar que las mismas impacten negativamente en las personas y en el medio ambiente.

A seguidas otro aspecto del presente artículo que reza: i) Fomentar la adecuada utilización de las fuentes de energía, propiciar la capacitación científica especializada sobre tecnologías de producción de energía eficiente.

Fundamentándose en los artículos aquí expuestos, se lleva a cabo la presente investigación con el propósito de sustentar el discurso escrito de los actores educativos inmersos en la misma.

\section{Senderos metodológicos}

«Pero entre más atrapados estamos por el mundo, más difícil nos es atraparlo. En la época de la telecomunicaciones, de la información, de la internet, estamos sumergidos por la complejidad del mundo, y las innumerables informaciones sobre el mundo ahogan nuestras posibilidades de inteligibilidad»

Edgar Morín.

Las representaciones sociales son la herramienta que permitirá develar los fundamentos explicativos de los co-participantes de la investigación, en relación a la educación tecnocientífica y su alfabetización en las ciencias básicas como medio para deconstruir y construir nuevos constructos del conocimiento científico. De este modo, las interpretaciones aquí concebidas se centran en una hermeneusis documental y del discurso escrito de los actores educativos del Departamento de Ciencias de la Universidad Nacional Pedro Henríquez Ureña (UNPHU), en Santo Domingo República Dominicana; a fin de comprender el fenómeno desde sus cosmovisiones. De tal manera, el análisis que se le da a esta investigación es de tipo cualitativo, debido a que la información se obtiene desde la interpretación de experiencias.

\section{Recorrido epistémico}

Esta investigación posee una naturaleza cualitativa de carácter descriptivo donde se amerita el uso del método fenomenológico hermenéutico, tal como se plantea en la corriente filosófica de 
Schütz (1979) y Gadamer (1999), al establecerse una relación con las implicaciones técnico-metodológicas para generar teoría, partiendo de fundamentos epistemológicos que ameritan: «dar cuenta de la realidad en estudio», desde la perspectiva de Gadamer, con la hermenéutica como filosofía.

Desde esta corriente, la interpretación es quién permite develar la realidad que se le atribuye al conocimiento de los actores sociales en el contexto educativo; desde sus normas, valores, percepciones y estilos de pensamiento. Por lo tanto, se debe estudiar los fundamentos explicativos de cada uno de ellos, desde la dialéctica existente entre la cosmovisión de quien interpreta el texto y el comportamiento del humano.

De tal manera que al pretender investigar sobre las representaciones sociales de la alfabetización tecnocientífica como una innovación desde el contexto universitario, se considera un proceso que emerge desde las necesidades sociales, culturales y académicas; que amerita el abordaje epistemológico donde se comprenda la realidad del sujeto y su conducta para conocer la visión que este posee del mundo. Partiendo de ello, este estudio se centra en el estudio fenomenológico-hermenéutico ya que parte de las experiencias, ideas, pensamientos y del «yo» en la construcción del conocimiento, tomando en cuenta que «se pueden tener experiencias comunes» (Husserl, 1992), permitiendo describir y comprender el mundo desde las experiencias vividas, haciendo énfasis en la interpretación de los significados del mundo (Lebenswelt s.f.) y el comportamiento de los actores sociales.

En el mismo orden de ideas se aborda la fenomenología como paradigma introspectivo vivencial, centrada en indagar la forma en que los actores educativos interpretan y comprenden sus experiencias y modo de conocer y aplicar la educación tecnocientífica desde la implementación de las ciencias básicas, dentro del contexto universitario, como el mundo vivido desde sus experiencias con su propio significado. Es elemental hacer énfasis en que la tecnociencia corresponde al auge de la cultura tecnológica en la sociedad del conocimiento, por lo tanto se genera una red de significados para el hombre y el entorno donde se desenvuelva.

\section{Contexto educativo}

El abordaje, comprensión, análisis e interpretación del mundo desde las experiencias vividas y el compartir cognitivo de las representaciones sociales de los sujetos, se llevará a cabo en el escenario del Departamento de Ciencias de la Universidad Nacional Pedro Henríquez Ureña (UNPHU), en Santo Domingo, República Dominicana, con la participación de tres (3) actores educativos conocidos como docentes.

\section{Al encuentro de la información (técnicas e instrumentos)}

La naturaleza de esta investigación es de carácter cualitativo, por tanto, la técnica usada se corresponde con el análisis documental. Esta permitió descomponer y describir los textos e investigaciones realizados referente al tema. A ella se sumó la entrevista a profundidad, que se apoya en el guion de entrevistas, a través de la cual se captaron la percepción, valores, imágenes y representaciones colectivas desde el conocimiento de los códigos de expresión así como las cristalizaciones ideológicas. (Galindo, 1998).

\section{Vías de procesamiento \\ de la información}

El procesamiento de la información se describe en la transcripción de las entrevistas ya aplicadas con la interpretación del discurso escrito de los actores sociales; obtenidas mediante las técnicas e instrumento de recolección de información. La información se visualizó a través de una matriz epistémica que Strauss y Corbin (2002; 200), definen como: «(...) una representación diagramática de un conjunto de ideas». En la misma se usan filtros epistémicos, categorizaciones y triangulaciones que dieron origen a la teorización de la investigación. 


\section{Validación de la información}

La técnica de validación de la información usada en la triangulación de la información consiste en cruzar la información recabada con la postura de autores, referente al tema y la interpretación del investigador, reinterpretando la situación en estudio a la luz de las evidencias provenientes de todas las fuentes empleadas en la investigación. Tiene como fin ofrecer credibilidad en los hallazgos. Para Denzin (1989) citado por Leal (2005; 16), se conoce como: «la combinación de dos o más teorías, fuentes de datos, método de investigación en el estudio de un fenómeno singular». Esto quiere decir, que es un proceso hermenéutico que parte de la opinión de los informantes, los autores o teóricos y el investigador, constituyendo los hallazgos imperantes al momento de una construcción teórica que emerge de las esencias propias de los sujetos de investigación. (Vergel; 2016).

\section{Teorización}

A través del discurso escrito de los actores educativos se pudo demostrar que la cultura tecnológica que se ha configurado en el contexto de la UNPHU, ha estado influenciada por las in-

\section{Referencias}

Farr, R. (1983). Escuelas Europeas de Psicología Social: La investigación de representaciones sociales en Francia. Revista Mexicana de Sociología. Año XLV Vol. XLV Instituto de Investigaciones Sociales. UNAM, pp.641-651.

Gadamer, H. (1977). Verdad y Método: Fundamentos de una hermenéutica filosófica. Salamanca.

__. (1999). ¿Quién soy yo y quién eres tú? Barcelona. España: Editorial Herder

Galindo, J. (1998). Técnicas de Investigación en Sociedad. Cultura y Comunicación. Consejo Nacional para la Cultura y las Artes. México: Adison Wesley Longman.

Gergen, K. (1996). Realidades y relaciones. Aproximaciones a la construcción social. Barcelona: Paidós.

Husserl, E. (1992). Invitación a la fenomenología. Barcelona: Paidós. novaciones del siglo XXI, como una necesidad de actualización social y académica para poder impartir procesos pedagógicos con el alcance que brindan las tecnologías. Adicionalmente se pudo interpretar que la cosmovisión que se posee del implemento de la cultura tecnológica en las ciencias básicas es compleja, pero a su vez necesaria, en cuanto a las demandas científicas, investigativas, metodológicas que exige la sociedad actualmente.

Asimismo, urge que las nociones de la realidad en el modo de concebir nuevos constructos sean interconectadas, para así poder desarrollar una educación tecnocientífica desde los saberes que se deconstruyen y construyen para que sean demostrados mediante el método científico. Funcionan como una muestra de nuevos descubrimientos que propician una evolución académica y por ende una innovación educativa. De este modo se fundamentan las representaciones sociales de los docentes que hacen vida en el Departamento de Ciencias de la UNPHU, en Santo Domingo, República Dominicana, con una necesidad imperante de educar tecnocientíficamente como lo exige el siglo XXI.

Latour, B. (1983). "Give Me a Laboratory and I will Raise the World», en Knorr-Cetina, K.D./Mulkay, M.J. (eds.) 1983. Science Observed: Perspectives on the Social Study of Science. London/Beverly Hills: Sage

Leal, J. (2005). La autonomía del sujeto investigador y la metodología de investigación. Universidad de los Andes. Mérida-Venezuela: Centro Editorial Litorama.

Ley 66-97. Ley General de Educación. República Dominicana. Recuperado de: http://www.oas.org/juridico/spanish/mesicic2_repdom_sc_anexo_7_sp.pdf

Moscovici, S. (1981). El fenómeno de las representaciones sociales en relaciones humanas, familia y sociedad. Buenos Aires, Argentina: Huemul S.A.

Mora, M. (2002). La Teoría de las Representaciones Sociales de Serge Moscovicci. Athenea Digital - Núm. 2. Otoño 2002. Universidad de Guadalajara México. Recuperado de: http://cort.as/-RvgM 
Pickering, A. (s.f.). Sciencie as Practice and Culture. The University of Chicago Press. Chicago.

Strauss, A. y Corbin, J. (2002). Bases de la Investigación Cualitativa. Técnicas y procedimientos para desarrollar la Teoría Fundamentada. Colombia: Universidad de Antioquia.

Sunkel, G. y Trucco, D. (2013). Las tecnologías digitales frente a los desafios de una educación inclusiva en América Latina. Santiago de Chile: Comisión Económica para América Latina y el Caribe (CEPAL).
Sunkel, G., Trucco, D. y Espejo, A. (2014). La integración de las tecnologías digitales en las escuelas de América Latina y el Caribe: una mirada multidimensional. Santiago de Chile: Comisión Económica para América Latina y el Caribe (CEPAL).

Vergel, E. (2016). Representaciones Sociales de la Cultura Tecnológica desde el Contexto Educativo Universitario: Imaginario Ignoto de la UPEL Maracay. [Tesis doctoral no publicada]. Universidad Pedagógica Experimental Libertador. Venezuela.

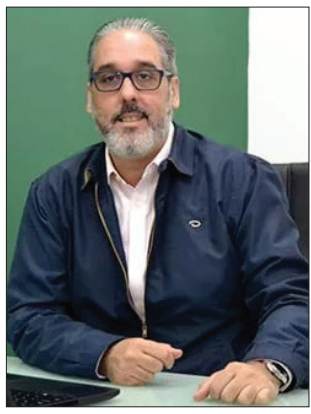

Emgelberth Vargas

Ingeniero Geofísico, de la Universidad Central de Venezuela; Máster en Gerencia de Proyectos y en Especialización en Cambio Climático y Doctor en Ciencias de la Educación por la Universidad Bicentenaria de Aragua, Venezuela. Labora en diferentes ramas del ámbito académico e investigación, con funciones de dirección, coordinación, apoyo académico, docencia e investigación, tanto en grado como en postgrado. Actualmente cursa el PhD en Física en la Universidad Central de Venezuela. 